OPEN ACCESS

Edited by:

Huali Wang,

Peking University Sixth Hospital,

China

Reviewed by:

Miguel A. Fernández-Blázquez,

Centro de Investigación

de Enfermedades Neurológicas

Foundation, Spain

Xiao Zhen LV

Peking University, China

*Correspondence:

Julius Popp

julius.popp@chuv.ch

Received: 30 November 2016 Accepted: 01 March 2017

Published: 14 March 2017

Citation:

Ouanes S, Castelao E,

von Gunten A, Vidal PM, Preisig M and Popp J (2017) Personality,

Cortisol, and Cognition in Non-demented Elderly Subjects:

Results from a Population-Based Study. Front. Aging Neurosci. 9:63. doi: 10.3389/fnagi.2017.00063

\section{Personality, Cortisol, and Cognition in Non-demented Elderly Subjects: Results from a Population-Based Study}

\author{
Sami Ouanes ${ }^{1}$, Enrique Castelao', Armin von Gunten', Pedro M. Vidal'2, Martin Preisig \\ and Julius Popp ${ }^{1 *}$

\begin{abstract}
${ }^{1}$ Department of Psychiatry, University Hospital of Lausanne, Lausanne, Switzerland, ${ }^{2}$ Department of Internal Medicine,
\end{abstract} \\ University Hospital of Lausanne, Lausanne, Switzerland
}

Certain personality traits, in particular higher neuroticism, have been associated, on one hand, with elevated cortisol levels, and on the other hand, with poorer cognitive performance. At the same time, several studies highlighted the association between high cortisol and poor cognitive functioning. Here, we hypothesized that increased cortisol may be associated with poorer cognition and with certain personality traits (mainly high neuroticism), and that personality might explain the association between cortisol and cognition. A cross-sectional analysis was conducted using data from Colaus/PsyColaus, a population-based study involving residents of Lausanne, Switzerland. Salivary cortisol samples (upon waking, 30 min after waking, at 11 am and at $8 \mathrm{pm}$ ) along with cognitive and personality measures were obtained from 643 non-demented participants aged at least 65. Personality traits were assessed using the NEO Five-Factor Inventory (NEOFFI). We examined the links between the cortisol Area under the Curve (AUC), the Clinical Dementia Rating Sum of Boxes (CDRSOB) and the NEO-FFI scores. No association was found between personality traits and the CDRSOB or the MMSE score, controlling for age, sex, depression, education and BMl. However, the executive functioning domain $z$-score was negatively associated with agreeableness $(p=0.005$; slope $=-0.107$ $[-0.181 ;-0.033])$ and openness $(p=0.029$; slope $=-0.081[-0.154 ;-0.008])$ after controlling for age, sex, depression, education and BMI. The CDRSOB score was positively associated with the cortisol AUC after controlling for age, sex, BMI, education and depression, $(p=0.003$; slope $=0.686$ [0.240; 1.333]). This association remained significant after controlling for personality traits and for the interaction between personality traits and the cortisol AUC $(p=0.006$; slope $=0.792$ [0.233; 1.352]. High agreeableness and openness might be associated with poorer executive performance in later life. Increased cortisol may be associated with both specific personality traits (high extraversion, low openness) and worse cognitive performance. Increased salivary cortisol does not mediate the relationship between personality traits and cognitive impairment.

Keywords: cognition, personality, cortisol, memory, dementia, neuroticism, agreeableness, openness 


\section{INTRODUCTION}

Certain personality traits (in particular neuroticism and to a lesser extent extraversion and openness) have been associated with Hypothalamus-Pituitary-Adrenal (HPA) axis dysregulation. Indeed, people with higher neuroticism tend to exhibit greater emotional responses to psychosocial stressors (Garcia-Banda et al., 2014). They also tend to have higher cortisol levels, likely reflecting a more pronounced stimulation of the HPA axis by psychosocial stressors (Portella et al., 2005; Yoshino et al., 2005; Gerritsen et al., 2009; Nater et al., 2010; Garcia-Banda et al., 2014; Miller et al., 2016).

However, other studies did not replicate this finding (Adler et al., 1997; Schommer et al., 1999; Ferguson, 2008) and some even reported a negative association between cortisol and neuroticism (Ballenger et al., 1983; LeBlanc and Ducharme, 2005).

Extraversion has also been associated with elevated cortisol plasma levels (Miller et al., 1999; LeBlanc and Ducharme, 2005), while agreeableness has been associated with lower salivary cortisol levels (Parent-Lamarche and Marchand, 2015).

Elevated cortisol levels have been associated with poorer cognitive performance in non-demented subjects (Lupien et al., 1994; Lee et al., 2008; Popp et al., 2009; Geerlings et al., 2015) as well as with more rapid disease progression in patients with Mild Cognitive Impairment (MCI) linked to Alzheimer's Disease (AD) (Csernansky et al., 2006; Popp et al., 2015).

Furthermore, some personality traits have been associated with poorer cognitive performance and even with an increased risk of dementia (von Gunten et al., 2009). Indeed, in older individuals, higher neuroticism has been associated with poorer cognitive performance independently of depression (Boyle et al., 2010), and in particular with poorer episodic memory (Wilson et al., 2004). High neuroticism and low conscientiousness were associated with greater decline in executive functions (Caselli et al., 2016). In a meta-analysis examining the link between specific facets of personality and the risk of $\mathrm{AD}$, individuals in the top quartile of neuroticism or the lowest quartile of conscientiousness had a threefold increased risk of incident $\mathrm{AD}$ (Terracciano et al., 2014).

Furthermore, previous studies examined the link between personality traits and either the HPA axis or the cognitive performance. As far as we know, no previous study scrutinized the inter-relationships between personality traits, the HPA axis and cognition.

In the light of these results highlighting that certain personality traits (higher neuroticism, in particular) might be associated with increased cortisol, that high cortisol might have deleterious cognitive effects, and that some personality traits might be associated with poorer cognition and increased risk for dementia, we hypothesized that personality traits might mediate the link between cortisol levels and cognitive impairment, in particular in episodic memory and executive functions.

\section{MATERIALS AND METHODS}

\section{Participants}

A cross-sectional analysis was conducted using data from the first follow-up of the longitudinal population-based CoLaus/PsyCoLaus study. The methodological features of this study were already described in detail (Firmann et al., 2008; Preisig et al., 2009). CoLaus/PsyColaus included a random sample of 6734 subjects (age range: 35-75 years) selected from the residents of the city of Lausanne, Switzerland, between 2003 and 2007. All subjects were invited to participate at the first follow-up, which took place between 2009 and 2013. A total of 4004 subjects accepted these new physical and psychiatric evaluations which also included salivary cortisol measures. In addition, all subjects aged 65 or older $(n=1918)$ were invited to undergo a neuropsychological assessment. Information on demographic, medical, and treatment history as well as smoking and alcohol consumption was gathered using semi-structured interviews conducted by trained interviewers or self-rating questionnaires. Psychiatric/behavioral disorders were assessed using the Diagnostic Interview for Genetic Studies (DIGS) (Preisig et al., 1999). Cardio-metabolic disorders were assessed clinically and with the use of biochemical measures. The distribution of age groups, sex and geographic distributions in CoLaus/Psycholaus participants at baseline were similar to the source population (Firmann et al., 2008).

\section{Cognitive Assessment}

A neuropsychological and clinical examination as well as an assessment of the participants' daily living activities were performed by trained master-level psychologists blinded for the salivary cortisol levels.

The neuropsychological assessment included:

- The assessment of the global cognitive performance using the Mini Mental State Examination (MMSE) (Folstein et al., 1975), the most commonly used screening tool for global cognitive impairment. MMSE scores range from 0 to 30, a higher score indicating better performance.

- The assessment of memory using the Grober and Buschke Double Memory Test (DMT) (Buschke et al., 1997).

- The assessment of verbal fluency using the DO40 picturenaming test (Deloche and Hannequin, 1997), the letter (phonemic) and the category (semantic) fluency tasks.

- The assessment of executive functions including cognitive flexibility, selective attention, cognitive inhibition, and information processing speed using the Stroop Test (Stroop, 1935).

- The assessment of visuo-spatial construction using the figures from the Consortium to Establish a Registry for Alzheimer's Disease (CERAD) neuropsychological test battery (Morris et al., 1988).

All of these tests and scales have been validated and are widely used in the field.

Overall cognitive and functional status was assessed using the Clinical Dementia Rating (CDR) scale, a widely used scale 
for the clinical staging of cognitive impairment. The CDR encompasses data about cognitive and functional performance in six domains: memory, orientation, judgment and problem solving, community affairs, home and hobbies, and personal care (Morris, 1993).

Participants with a global $\mathrm{CDR}>=1$ (defining dementia) were excluded from the current study.

The CDR Sum of Boxes (CDRSOB) was calculated.

\section{Personality Assessment}

Personality traits were measured using the Revised NEO FiveFactor Inventory (NEO-FFI-R) in its French version validated in Switzerland (Aluja et al., 2005). The NEO-FFI-R is the short version of the revised NEO Personality Inventory (NEO-PI-R). The questionnaire measures the five main personality dimensions of the Five-Factor Model, i.e., neuroticism, extraversion, openness to experience, agreeableness, and conscientiousness. Participants were asked to respond to 60 items using a 5-point Likert scale ranging from 1 (strongly disagree) to 5 (strongly agree). For each of the five factors, a score is obtained by summing up the scores of the corresponding items (Costa, 1994).

\section{Cortisol Measures}

Salivary cortisol has been established as a reliable indicator of circulating cortisol levels and HPA axis function (Gallagher et al., 2006). Participants used Salivette sampling devices (Sarstedt, Rommelsdorf, Germany) for saliva collection.

Four salivary samples were obtained from each participant: upon waking, $30 \mathrm{~min}$ after waking, at 11 am and at $8 \mathrm{pm}$. Subjects were instructed not to brush their teeth and to refrain from eating, drinking, smoking, and exercising $30 \mathrm{~min}$ prior to and during the self-administered sampling procedure (Kuehner et al., 2007). Subjects were also instructed to keep a saliva collection sheet where they would record adherence to the protocol including exact time of saliva collections. Until sampling had been completed, subjects stored the saliva samples at home in their freezers before returning them to the laboratory together with the saliva protocol, where they were stored at $-20^{\circ} \mathrm{C}$ until biochemical analysis.

Free cortisol levels in the salivary samples were measured using a commercially available chemiluminescence assay (IBL, Hamburg, Germany). Inter- and intra-assay coefficients of variability were $<9 \%$.

In case of non-adherence to the protocol, missing values were analyzed using the expectation-maximization algorithm.

We calculated cortisol Area Under the Curve (AUC) using the trapezoid formula (Pruessner et al., 2003). The AUC is a commonly used measure to estimate total hormonal output over a period of time. Unlike other summary measures such as mean cortisol, the AUC captures not only the cortisol levels at the times of sampling but also changes over time (Pruessner et al., 2003).

We also calculated the cortisol awakening response (CAR) defined as the difference between cortisol 30 min after waking and cortisol upon waking.

\section{Other Variables}

Subjective cognitive decline was assessed using the Cognitive Complaint Questionnaire (QPC) (Thomas-Antérion et al., 2003). The QPC is a French-language rater-administered instrument consisting of 10 yes/no questions assessing subjective cognitive changes over the previous 6 months. According to the scoring method proposed by the authors, subjective cognitive decline is considered present if the participant gave at least three positive answers to the 10 questions and/or a positive answer to question 5 and/or at least two positive answers to questions A,4,5,7,8.

Depressive symptoms were assessed at physical evaluation (approximately 1 year before the cognitive assessment) using the Center for Epidemiologic Studies Depression scale (CES-D) in its French version (Morin et al., 2011). CES-D scores range between 0 and 60 with higher scores indicating more depressive symptoms. Depressive symptoms were clinically re-assessed at the same time as the cognitive assessment.

Level of formal education was categorized into two groups: primary/secondary school education and higher education.

Body Mass Index (BMI) was also included as a covariate since cortisol levels might depend on BMI (Odeniyi et al., 2015).

\section{Ethics Statement}

The CoLaus/PsyCoLaus study was approved by the Ethics Committee of the University of Lausanne and written informed consent was obtained from all participants.

\section{Statistical Analysis}

Statistical analysis was performed using SPSS v23.0 (IBM Corp., Armonk, NY, USA).

\section{Descriptive Statistics}

Continuous variables were described as mean \pm standard deviation, while categorical variables were described through absolute and relative frequencies.

The scores of each cognitive domain (memory, fluency, executive functions and visuo-spatial construction) were standardized ( $z$-scores were calculated for the sum of each cognitive domain score).

\section{Associations between Cognitive Performance and Cortisol}

Hierarchical multiple linear regression models were constructed with the MMSE score as an outcome variable and with the cortisol AUC then the CAR (respectively) as independent variables, controlling for age, sex, BMI, education and CES-D score.

Afterward, each cognitive domain $z$-score was considered as a dependant variable with cortisol AUC then CAR (respectively) as independent variables, controlling for age, sex, BMI, education and CES-D score.

\section{Associations between Cognitive Performance and Personality Traits}

Hierarchical multiple logistic regression models were constructed with the MMSE score, then with the CDRSOB and each cognitive domain $z$-score respectively as dependent variables, and with the 
NEO-FFI-R trait scores as independent variables, controlling for age, sex, BMI, education and CES-D score.

\section{Associations between Cortisol and Personality Traits}

Hierarchical multiple logistic regression models were constructed with the cortisol AUC then with the CAR respectively as dependent variables, and with the NEO-FFI-R trait scores as independent variables, controlling for age, sex, BMI, education and CES-D score.

\section{Associations between Cognitive Performance, Cortisol and Personality Traits}

A three-block hierarchical multiple regression model was constructed with the MMSE score as a dependant variable. Block one included age, sex, BMI, education and the CES-D score. Block two included the NEO-FFI-R trait scores and the interaction between NEO-FFI-R trait scores and the cortisol AUC and block three the cortisol AUC.

For each of these regression models, the unstandardized regression coefficients (B), their 95\% confidence intervals (CIs), the corresponding $\mathrm{R}$ squares $\left(R^{2}\right)$ and the $p$-values are presented.

The mediation hypothesis was assessed using Sobel's method (Sobel, 1982).

For multiple comparisons, $p$-values were adjusted according to Holm-Bonferroni's method.

\section{RESULTS}

\section{Characteristics of the Sample}

Of the 1918 subjects aged at least 65 at the first follow-up visit of CoLaus/PsyCoLaus, 1214 had a thorough cognitive assessment and 643 non-demented (CDR score $<1$ ) individuals also agreed to provide salivary samples and underwent a personality assessment (33.5\% of all participants in this age range) (Figure 1).

Compared with the whole PsyCoLaus sample aged at least 65, our sub-sample had a comparable distribution by age, gender, education level, BMI and CES-D score (Table 1).

Among the salivary cortisol measures, 164 (6.4\% of the total number of values) were missing.

The results of the different cognitive scores, the personality assessment as well as the cortisol measures are described in Table 2.

Subjective cognitive decline was not associated with cortisol, cognition, depression or personality traits, and was not included in further statistical analysis.

Table 3 shows the Spearman's correlations between the Big Five traits in our population. Although the correlations were significant, they were modest, and induced no multicollinearity issues in regression models as checked by tolerance.

\section{Personality Traits and Cortisol}

Cortisol AUC was associated negatively with extraversion $(p=0.005$; slope $=-2.850[-4.854 ;-0.846])$ and positively with openness $\left(p=0.008\right.$; slope $\left.=2.412[0.628 ; 4.197] ; R^{2}=0.030\right)$ after controlling for age, sex, depressive symptoms and BMI.

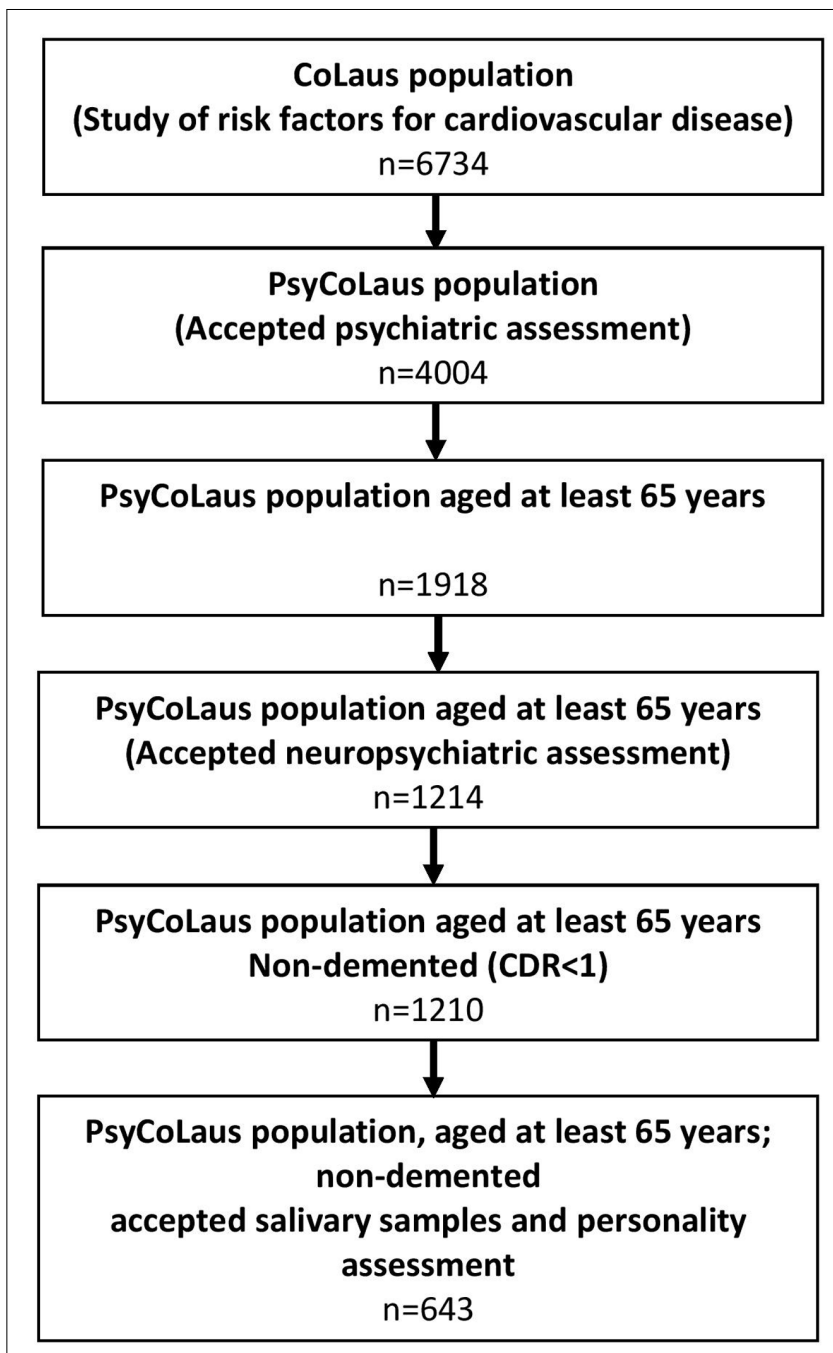

FIGURE 1 | Flow chart of the included participants.

TABLE 1 | General characteristics of the studied samples.

\begin{tabular}{lccc}
\hline & $\begin{array}{c}\text { PsyCoLaus } \\
\text { sample aged at } \\
\text { least } \mathbf{6 5}\end{array}$ & Studied sample & $\boldsymbol{p}$ \\
\hline$n$ & 1214 & & \\
Age, years (m \pm SD) & $71.6 \pm 4.7$ & $71.5 \pm 4.5$ & 0.66 \\
Gender, \% women & 57.9 & 57.7 & 0.93 \\
Education level, \% & 40.5 & 38.3 & 0.36 \\
higher education & $26.9 \pm 4.7$ & $26.7 \pm 4.5$ & 0.38 \\
BMl, Kg/m² (m \pm SD) & $10.3 \pm 8.2$ & $10.0 \pm 7.9$ & 0.45 \\
CES-D score (m \pm SD) & & & \\
\hline$m$, mean; SD, standard deviation; BMI, Body Mass Index; CES-D, Center for \\
Epidemiologic Studies Depression Scale.
\end{tabular}

Cortisol awakening response was negatively associated with the neuroticism score $(p=0.048$; slope $=-1.117[-2.222$; $\left.-0.011] ; R^{2}=0.031\right)$ after controlling for age, sex, depression, education and BMI. 
TABLE 2 | Cognitive, personality and cortisol profile of the studied population.

\begin{tabular}{|c|c|}
\hline MMSE score (0-30) (m $\pm S D)$ & $29.2 \pm 1.6$ \\
\hline CDRSOB median (P25;P75) & $0.5(1.0 ; 1.5)$ \\
\hline \multicolumn{2}{|l|}{ CDR score, $\mathrm{n}(\%)$} \\
\hline 0 & $291(45.3)$ \\
\hline 0.5 & $352(54.7)$ \\
\hline QPC, subjective cognitive decline present, $n(\%)$ & $42(21.0)$ \\
\hline \multicolumn{2}{|l|}{$\mathrm{DMT}(\mathrm{m} \pm S D)$} \\
\hline Immediate recall (0-16) & $15.8 \pm 1.1$ \\
\hline Total free recall (0-48) & $29.8 \pm 6.9$ \\
\hline Total cued recall $(0-48)^{*}$ & $18.0 \pm 9.2$ \\
\hline Identification (0-16) & $15.9 \pm 0.4$ \\
\hline Recognition (0-48) & $44.8 \pm 9.4$ \\
\hline Delayed free recall $(0-16)$ & $11.6 \pm 2.6$ \\
\hline Delayed cued recall $(0-16)^{*}$ & $4.9 \pm 3.7$ \\
\hline DO40 picture-naming test $(0-40)(\mathrm{m} \pm S D)$ & $39.7 \pm 1.2$ \\
\hline Semantic verbal fluency $(\mathrm{m} \pm S D)$ & $29.5 \pm 8.1$ \\
\hline Phonemic verbal fluency $(\mathrm{m} \pm S D$ ) & $20.9 \pm 7.8$ \\
\hline Stroop test, correct items $(\mathrm{m} \pm S D$ ) & $23.9 \pm 0.6$ \\
\hline Stroop dots condition (0-24) & $23.9 \pm 0.6$ \\
\hline Stroop words condition (0-24) & $23.9 \pm 0.4$ \\
\hline Stroop interference condition (0-24) & $23.2 \pm 1.9$ \\
\hline CERAD figures $(0-11)(m \pm S D)$ & $10.5 \pm 1.0$ \\
\hline \multicolumn{2}{|l|}{$\mathrm{NEO}-\mathrm{FFI}$} \\
\hline Neuroticism (0-48) $(m \pm S D)$ & $17.3 \pm 6.7$ \\
\hline Extraversion $(0-48)(m \pm S D)$ & $27.3 \pm 6.0$ \\
\hline Openness (0-48) $(m \pm S D)$ & $29.0 \pm 5.6$ \\
\hline Agreeableness (0-48) $(m \pm S D)$ & $33.8 \pm 5.1$ \\
\hline Consciousness (0-48) $(\mathrm{m} \pm S D)$ & $34.6 \pm 5.4$ \\
\hline Cortisol AUC, in $\mu \mathrm{mol} . h / L(m \pm S D)$ & $0.28 \pm 0.11$ \\
\hline CAR, in nmol/L, median (P25;P75) & $6.3(-0.5 ; 12.7)$ \\
\hline
\end{tabular}

m, mean; SD, standard deviation; CDR, Clinical Dementia Rating; CDRSOB, Clinical Dementia Rating Sum Of Boxes; QPC, Cognitive Complaint Questionnaire; MMSE, Mini Mental State Examination; DMT, Grober and Buschke Double Memory Test; DO40: CERAD, Consortium to Establish a Registry for Alzheimer's Disease; NEO Five-Factor Inventory; AUC, Area Under the Curve; CAR, Cortisol Awakening Response.

* Cued recall scores refer to the number of additional items recalled with cues after the free recall step.

\section{Personality Traits and Cognitive Performance}

No association was found between personality traits and the CDRSOB or the MMSE score, controlling for age, sex, depression, education and BMI.

Among the cognitive domains, only the executive functioning domain $z$-score was negatively associated with agreeableness $(p=0.005 ;$ slope $=-0.107[-0.181 ;-0.033])$ and openness $\left(p=0.029\right.$; slope $\left.=-0.081[-0.154 ;-0.008] ; R^{2}=0.107\right)$ after controlling for age, sex, depression, education and BMI.

\section{Cognitive Performance and Cortisol}

The CDRSOB was positively associated with the cortisol AUC after controlling for age, sex, BMI, education and depressive symptoms $(p=0.003$; slope $=0.686[0.240 ; 1.333]$; $R^{2}=0.089$ ) (Figure 2). Similarly, the MMSE score was negatively associated with the cortisol AUC, after controlling for age, sex, BMI, education and depressive symptoms (regression $p=0.039 ;$ slope $\left.=-1.079[-2.106 ;-0.052] ; R^{2}=-0.039\right)$ These associations became not significant, however, when the individuals with a cortisol AUC above the 95th percentile are excluded.

Among the cognitive domains, only the memory domain $z$-score was significantly associated with cortisol AUC $(p=0.025$; slope $\left.=-0.791[-1.485 ;-0.098] ; R^{2}=0.083\right)$. Neither the MMSE score nor any of the cognitive domain $z$-scores was associated with the CAR.

\section{Are Personality Traits Related to the Cortisol AUC Thereby Affecting Global Cognitive Performance?}

The CDRSOB score was positively associated with the cortisol AUC after controlling for age, sex, BMI, education and depression, $\left(p=0.003\right.$; slope $\left.=0.686[0.240 ; 1.333] ; R^{2}=0.089\right)$. This association remained significant after controlling for personality traits and for the interaction between personality traits and the cortisol AUC $(p=0.006$; slope $=0.792[0.233$; 1.352]; $R^{2}=0.107$ ) (Figure 2). Sobel test for mediation was not significant.

Post hoc power analysis found an observed statistical power of 0.993.

\section{DISCUSSION}

We analyzed the relationships between personality traits, cortisol levels and cognitive performance in a large sample of nondemented subjects aged 65 years or more from the general population. While higher cortisol was associated both with some traits (low extraversion and high openness) and with poorer cognition, our results suggest that cortisol does not mediate the relationship between personality traits and cognition.

\section{Personality Traits and Cortisol}

In our study, individuals with higher openness tended to have higher cortisol levels. This finding is in line with Bibbey et al.'s (2013) study which showed that participants who were less open had smaller cortisol reaction to stress. Yet, other studies did not find any association between openness and cortisol (LeBlanc and Ducharme, 2005; Gerritsen et al., 2009; van Santen et al., 2011). This discrepancy is likely explained by different studied populations.

Lower extraversion was associated with higher cortisol levels. Since extraversion is associated with warmth, assertiveness and positive emotions (LeBlanc and Ducharme, 2005), we might expect extravert individuals to deal better with stress and thus to have higher thresholds to activate their HPA axis. However, previous studies either showed an opposite tendency (higher extraversion associated with higher cortisol) (Miller et al., 1999; LeBlanc and Ducharme, 2005), or found no significant association (Ballenger et al., 1983; Schommer et al., 1999).

We did not find any link between neuroticism and cortisol. While this result is similar to that of some other studies (Adler 
TABLE 3 | Spearman correlations between the Big Five traits in the studied population.

\begin{tabular}{|c|c|c|c|c|c|c|}
\hline & & Neuroticism & Extraversion & Openness & Agreeableness & Conscientiousness \\
\hline Neuroticism & $\rho^{*} p$ & - & $-0.391<0.001$ & $-0.165<0.001$ & $-0.276<0.001$ & $-0.401<0.001$ \\
\hline Extraversion & $\rho^{*} p$ & & - & $0.271<0.001$ & $0.244<0.001$ & $0.426<0.001$ \\
\hline Openness & $\rho^{*} p$ & & & - & $0.178<0.001$ & $0.171<0.001$ \\
\hline Agreeableness & $\rho^{*} p$ & & & & - & $0.320<0.001$ \\
\hline
\end{tabular}

* $\rho$, Spearman's correlation coefficient.
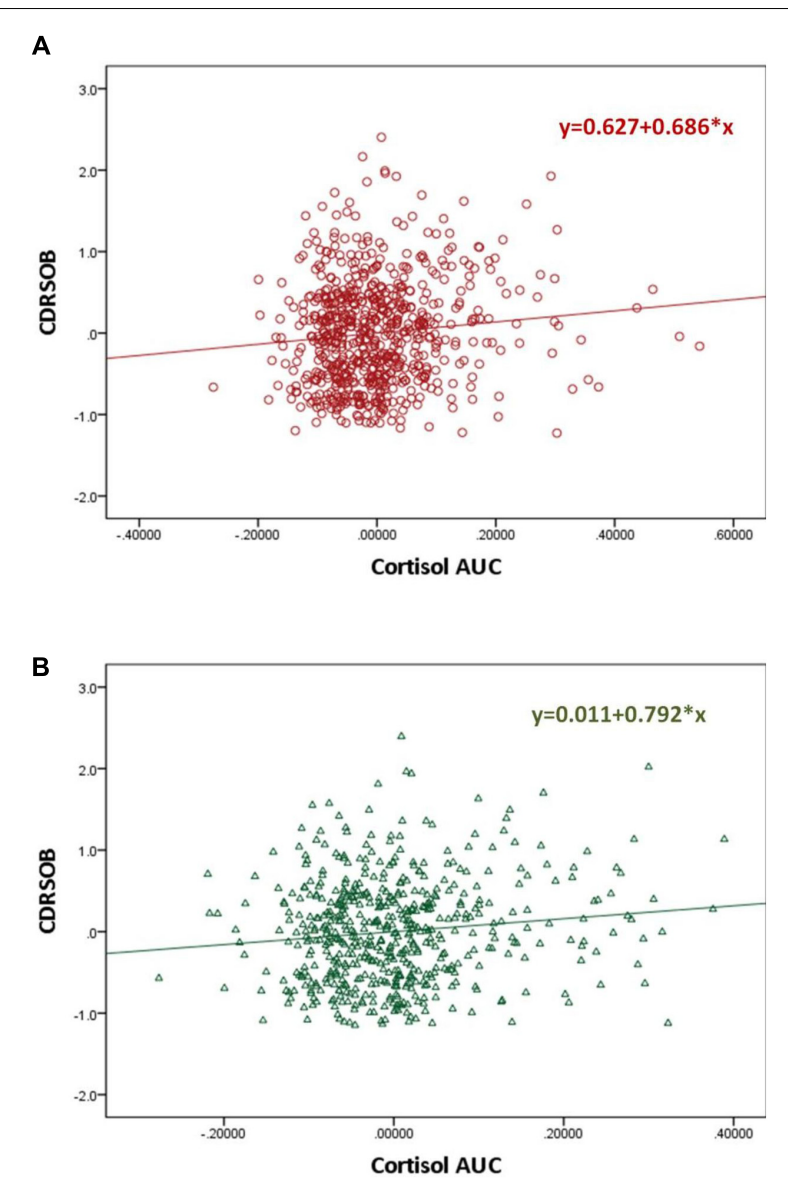

FIGURE 2 | Scatter plot graphs of partial regression analysis result for the association between the MMSE and Cortisol Area Under the Curve before (A) and after (B) controlling for personality Big Five traits and the interaction between Big Five traits and the cortisol AUC*

et al., 1997; Schommer et al., 1999; Ferguson, 2008), neuroticism has often been associated with elevated cortisol (Bridges and Jones, 1968; van Eck et al., 1996; Miller et al., 1999; Portella et al., 2005; Yoshino et al., 2005; Gerritsen et al., 2009; Nater et al., 2010; Garcia-Banda et al., 2014; Miller et al., 2016), yet also with decreased cortisol (Ballenger et al., 1983; LeBlanc and Ducharme, 2005).

This discrepancy is probably due to different studied populations and to the effects of depression and anxiety which have been controlled for in some studies but not in others.

\section{Personality Traits and Cognitive Performance}

We did not find any relationship between the MMSE score or the CDRSOB and personality traits. In our study, we considered all of the Big Five factors, whereas most prior studies examining the link between cognition and personality traits focused solely on neuroticism and a few additionally examined extraversion.

Higher neuroticism has been reported to be associated with lower MMSE scores in older primary care patients independently of depression (Boyle et al., 2010) as well as community-dwelling individuals aged at least 70 years independently of depression and anxiety (Jorm et al., 1993). This association might be stronger in demented subjects but seems to be also present among non-demented individuals (Jorm et al., 1993). Higher neuroticism seems to be associated with poorer episodic memory in community-dwelling individuals (Jorm et al., 1993; Meier et al., 2002; Klaming et al., 2016) as well as in patients with AD (Wilson et al., 2004). Higher neuroticism has been shown to be linked to an increased risk of incident AD dementia (Terracciano et al., 2014).

Data about the association between extraversion and cognitive measures have been scarce and discrepant. Indeed, Meier et al. found that extraversion positively correlated with episodic memory performance (Meier et al., 2002). This link was explained by more extraverted older adults being probably more stimulated and less affected by the loss of social interactions that occurs with age (Meier et al., 2002). However, others did not find any link between cognition and extraversion (Jorm et al., 1993).

A meta-analysis examining personality traits and the risk for $\mathrm{AD}$ showed that higher agreeableness could be a protective factor against AD (Terracciano et al., 2014).

Moreover, since agreeableness is characterized by altruism and kindheartedness, agreeable people might be more inclined to deal positively with stressors. Hence, individuals with low agreeableness are probably more prone to react negatively to stressors, in the same way as people with high neuroticism, thus explaining our finding (Parent-Lamarche and Marchand, 2015).

We found that individuals scoring higher on agreeableness or openness had poorer executive functioning. This result is in line with Ikeda et al.'s (2014) study reporting an association between higher agreeableness scores and weakened suppression of the default mode network. This network consists of the posterior cingulate cortex and precuneus, the inferior parietal cortices, and dorsal and ventral areas of the medial frontal cortex and is normally suppressed during frontal tasks (Ikeda et al., 2014). 
Finally, we did not find any association between conscientiousness and cognition, while a clinical and autopsy study, found higher premorbid conscientiousness in demented patients with cerebral AD pathology compared to non-demented individuals with cerebral AD pathology (Terracciano et al., 2014).

\section{Are Personality Traits Related to the Cortisol AUC Thereby Affecting Global Cognitive Performance?}

Specific personality traits might alter cognition and/or increase the risk for $\mathrm{AD}$ through increased cortisol output. Indeed, as personality traits affect the way individuals cope with stressors they might modulate the effects of the various stressors over the lifespan on the HPA axis. At the same time, elevated cortisol has commonly been associated with cognitive impairment (Lupien et al., 2007; Lee et al., 2008; Tatomir et al., 2014; Geerlings et al., 2015; Vogel et al., 2016), hippocampal atrophy (Sapolsky, 2000; Tatomir et al., 2014) and AD pathology (Green et al., 2006).

Our findings suggest that personality traits might not mediate or modify the association between cortisol and cognitive performance. Certain personality traits might lead to poorer cognitive performance, independently of cortisol, through other mechanisms including higher rates of cigarette smoking, physical inactivity, obesity and depression which in turn are known risk factors for dementia, as well as effects on inflammatory markers (particularly, IL6, CRP and leucocyte count) and neurotrophic factors. Furthermore, shared genetic liability (involving the DYRK1A and the APOE genes) has also been suggested as a possible mechanism for the association between specific personality traits and AD pathology (Terracciano et al., 2014).

The association we found between cortisol and cognitive performance can be explained by factors other than stress increasing cortisol output more or less depending on the personality traits. Indeed, increased cortisol is associated with the metabolic syndrome, which is tightly linked to vascular dementia and AD (Kim and Feldman, 2015). Moreover, since the hippocampus normally inhibits the HPA axis, the hippocampal atrophy associated with $\mathrm{AD}$ might promote the cortisol release (Geerlings et al., 2015).

\section{Strengths and Limitations}

Major strengths of this study include its population-based design, the large sample size for this type of studies (many other studies had fewer than 100 participants) as well as the detailed neuropsychological examination. While many other studies only examined one or two personality traits, mainly neuroticism, we assessed the Big Five personality traits. A previous personality assessment allowed us to disentangle effects of pre-morbid personality traits from personality changes associated with cognitive decline.

Nevertheless, some limitations are to be acknowledged. First, the cross-sectional design does not allow us to draw any conclusions about cause-to-effect relationships. Second, only
$33.5 \%$ of the participants of 65 years and older underwent both the cognitive and the personality assessments and the cortisol measures. However, the included sub-sample had a comparable distribution by age, gender, education level, BMI and CESD score with the whole PsyCoLaus sample aged at least 65 . Third, since the assessment of personality traits was crosssectional, it does not allow us to draw any conclusions on whether the measured traits were the "stable" adult traits or the traits already modified by a potential neurodegenerative process. Indeed, personality changes seem to occur early in the course of neurocognitive disorders, at the stage of MCI (Donati et al., 2013), although these changes seem to remain moderate. Fourth, we did not collect information about perceived stress at the moment of the study. Different levels of perceived stress might have affected the cortisol levels.

\section{CONCLUSION}

High agreeableness and openness might be associated with poorer executive performance. High extraversion and low openness might be associated with increased cortisol. The association between personality traits and cognitive impairment seems to be independent of increased cortisol production and its effects on cognition. Hence, specific personality traits may influence cognition through other mechanisms. According to other studies, these mechanisms may include effects on lifestyle and health related behavior including smoking, dietary habits and physical activity with consequences for cardiovascular risk and the production of cytokines and neurotrophic factors.

\section{AUTHOR CONTRIBUTIONS}

Conception and design of the study: SO, EC, AvG, PV, MP, and JP. Data analysis/interpretation: SO, EC, MP, and JP. Drafting the article: SO and JP. Revising the article: SO, EC, AvG, PV, $\mathrm{MP}$, and JP.

\section{FUNDING}

The CoLaus/PsyCoLaus study was and is supported by research grants from GlaxoSmithKline, the Faculty of Biology and Medicine of Lausanne, and the Swiss National Research Foundation (grants 3200B0-105993, 3200B0-118308, 33CSCO122661, 33CS30-139468, and 33CS30-148401). JP received support from the Swiss National Research Foundation (grant 320030-141179).

\section{ACKNOWLEDGMENTS}

The authors would like to express their gratitude to the Lausanne inhabitants who volunteered to participate in the PsyCoLaus study. We would also like to thank all the investigators of the CoLaus study, which made the psychiatric PsyCoLaus study possible. 


\section{REFERENCES}

Adler, L., Wedekind, D., Pilz, J., Weniger, G., and Huether, G. (1997). Endocrine correlates of personality traits: a comparison between emotionally stable and emotionally labile healthy young men. Neuropsychobiology 35, 205-210. doi: $10.1159 / 000119346$

Aluja, A., García, O., Rossier, J., and García, L. F. (2005). Comparison of the NEO-FFI, the NEO-FFI-R and an alternative short version of the NEO-PIR (NEO-60) in Swiss and Spanish samples. Pers. Individ. Dif. 38, 591-604. doi: 10.1016/j.paid.2004.05.014

Ballenger, J. C., Post, R. M., Jimerson, D. C., Lake, C. R., Murphy, D., Zuckerman, M., et al. (1983). Biochemical correlates of personality traits in normals: an exploratory study. Pers. Individ. Dif. 4, 615-625. doi: 10.1016/01918869(83)90116-2

Bibbey, A., Carroll, D., Roseboom, T. J., Phillips, A. C., and de Rooij, S. R. (2013). Personality and physiological reactions to acute psychological stress. Int. J. Psychophysiol. 90, 28-36. doi: 10.1016/j.ijpsycho.2012.10.018

Boyle, L. L., Lyness, J. M., Duberstein, P. R., Karuza, J., King, D. A., Messing, S., et al. (2010). Trait neuroticism, depression, and cognitive function in older primary care patients. Am. J. Geriatr. Psychiatry 18, 305-312. doi: 10.1097/JGP. 0b013e3181c2941b

Bridges, P. K., and Jones, M. T. (1968). Relationship of personality and physique to plasma cortisol levels in response to anxiety. J. Neurol. Neurosurg. Psychiatry 31, 57-60. doi: 10.1136/jnnp.31.1.57

Buschke, H., Sliwinski, M. J., Kuslansky, G., and Lipton, R. B. (1997). Diagnosis of early dementia by the double memory test: encoding specificity improves diagnostic sensitivity and specificity. Neurology 48, 989-997. doi: 10.1212/ WNL.48.4.989

Caselli, R. J., Dueck, A. C., Locke, D. E., Henslin, B. R., Johnson, T. A., Woodruff, B. K., et al. (2016). Impact of personality on cognitive aging: a prospective cohort study. J. Int. Neuropsychol. Soc. 22, 765-776. doi: 10.1017/ S1355617716000527

Costa, P. (1994). Revised Neo Personality Inventory (Neo Pi-R) and Neo Five-factor Inventory (Neo-Ffi): Professional Manual. Lutz, FL: Psychological Assessment Resources.

Csernansky, J. G., Dong, H., Fagan, A. M., Wang, L., Xiong, C., Holtzman, D. M., et al. (2006). Plasma cortisol and progression of dementia in subjects with Alzheimer-type dementia. Am. J. Psychiatry 163, 2164-2169. doi: 10.1176/ajp. 2006.163.12.2164

Deloche, G., and Hannequin, D. (1997). DO 80: Epreuve de Dénomination Orale dimages. Pensacola, FL: Centre de Psychologie Appliquée.

Donati, A., Studer, J., Petrillo, S., Pocnet, C., Popp, J., Rossier, J., et al. (2013). The evolution of personality in patients with mild cognitive impairment. Dement. Geriatr. Cogn. Disord. 36, 329-339. doi: 10.1159/000353895

Ferguson, E. (2008). Health anxiety moderates the daytime cortisol slope. J. Psychosom. Res. 64, 487-494. doi: 10.1016/j.jpsychores.2008. 01.011

Firmann, M., Mayor, V., Vidal, P. M., Bochud, M., Pecoud, A., Hayoz, D., et al. (2008). The CoLaus study: a population-based study to investigate the epidemiology and genetic determinants of cardiovascular risk factors and metabolic syndrome. BMC Cardiovasc. Disord. 8:6. doi: 10.1186/14712261-8-6

Folstein, M. F., Folstein, S. E., and McHugh, P. R. (1975). Mini-mental state. A practical method for grading the cognitive state of patients for the clinician. J. Psychiatr. Res. 12, 189-198. doi: 10.1016/0022-3956(75)90026-6

Gallagher, P., Leitch, M. M., Massey, A. E., McAllister-Williams, R. H., and Young, A. H. (2006). Assessing cortisol and dehydroepiandrosterone (DHEA) in saliva: effects of collection method. J. Psychopharmacol. 20, 643-649. doi: 10.1177/ 0269881106060585

Garcia-Banda, G., Chellew, K., Fornes, J., Perez, G., Servera, M., and Evans, P. (2014). Neuroticism and cortisol: pinning down an expected effect. Int. J. Psychophysiol. 91, 132-138. doi: 10.1016/j.ijpsycho.2013.12.005

Geerlings, M. I., Sigurdsson, S., Eiriksdottir, G., Garcia, M. E., Harris, T. B., Gudnason, V., et al. (2015). Salivary cortisol, brain volumes, and cognition in community-dwelling elderly without dementia. Neurology 85, 976-983. doi: 10.1212/WNL.0000000000001931

Gerritsen, L., Geerlings, M. I., Bremmer, M. A., Beekman, A. T., Deeg, D. J., Penninx, B. W., et al. (2009). Personality characteristics and hypothalamic-pituitary-adrenal axis regulation in older persons. Am. J. Geriatr. Psychiatry 17, 1077-1084. doi: 10.1097/JGP.0b013e3181bd1be6

Green, K. N., Billings, L. M., Roozendaal, B., McGaugh, J. L., and LaFerla, F. M. (2006). Glucocorticoids increase amyloid-beta and tau pathology in a mouse model of Alzheimer's disease. J. Neurosci. 26, 9047-9056. doi: 10.1523/ JNEUROSCI.2797-06.2006

Ikeda, H., Ikeda, E., Shiozaki, K., and Hirayasu, Y. (2014). Association of the fivefactor personality model with prefrontal activation during frontal lobe task performance using two-channel near-infrared spectroscopy. Psychiatry Clin. Neurosci. 68, 752-758. doi: 10.1111/pcn.12190

Jorm, A. F., Mackinnon, A. J., Christensen, H., Henderson, S., Scott, R., and Korten, A. (1993). Cognitive functioning and neuroticism in an elderly community sample. Pers. Individ. Dif. 15, 721-723. doi: 10.1016/0191-8869(93) 90013-S

Kim, B., and Feldman, E. L. (2015). Insulin resistance as a key link for the increased risk of cognitive impairment in the metabolic syndrome. Exp. Mol. Med. 47, e149. doi: $10.1038 / \mathrm{emm} .2015 .3$

Klaming, R., Veltman, D. J., and Comijs, H. C. (2016). The impact of personality on memory function in older adults-results from the Longitudinal Aging Study Amsterdam. Int. J. Geriatr. Psychiatry doi: 10.1002/gps.4527 [Epub ahead of print].

Kuehner, C., Holzhauer, S., and Huffziger, S. (2007). Decreased cortisol response to awakening is associated with cognitive vulnerability to depression in a nonclinical sample of young adults. Psychoneuroendocrinology 32, 199-209. doi: 10.1016/j.psyneuen.2006.12.007

LeBlanc, J., and Ducharme, M. B. (2005). Influence of personality traits on plasma levels of cortisol and cholesterol. Physiol. Behav. 84, 677-680. doi: 10.1016/j. physbeh.2005.02.020

Lee, B. K., Glass, T. A., Wand, G. S., McAtee, M. J., Bandeen-Roche, K. Bolla, K. I., et al. (2008). Apolipoprotein e genotype, cortisol, and cognitive function in community-dwelling older adults. Am. J. Psychiatry 165, 1456-1464. doi: 10.1176/appi.ajp.2008.07091532

Lupien, S., Lecours, A. R., Lussier, I., Schwartz, G., Nair, N. P., and Meaney, M. J. (1994). Basal cortisol levels and cognitive deficits in human aging. J. Neurosci. 14(5 Pt 1), 2893-2903.

Lupien, S. J., Maheu, F., Tu, M., Fiocco, A., and Schramek, T. E. (2007). The effects of stress and stress hormones on human cognition: implications for the field of brain and cognition. Brain Cogn. 65, 209-237. doi: 10.1016/j.bandc.2007.02.007

Meier, B., Perrig-Chiello, P., and Perrig, W. (2002). Personality and memory in old age. Aging Neuropsychol. Cogn. 9, 135-144. doi: 10.1076/anec.9.2.135.9544

Miller, G. E., Cohen, S., Rabin, B. S., Skoner, D. P., and Doyle, W. J. (1999). Personality and tonic cardiovascular, neuroendocrine, and immune parameters. Brain Behav. Immun. 13, 109-123. doi: 10.1006/brbi.1998.0545

Miller, K. G., Wright, A. G., Peterson, L. M., Kamarck, T. W., Anderson, B. A., Kirschbaum, C., et al. (2016). Trait positive and negative emotionality differentially associate with diurnal cortisol activity. Psychoneuroendocrinology 68, 177-185. doi: 10.1016/j.psyneuen.2016.03.004

Morin, A. J., Moullec, G., Maiano, C., Layet, L., Just, J. L., and Ninot, G. (2011). Psychometric properties of the Center for Epidemiologic Studies Depression Scale (CES-D) in French clinical and nonclinical adults. Rev. Epidemiol. Sante Publique 59, 327-340. doi: 10.1016/j.respe.2011.03.061

Morris, J. C. (1993). The Clinical Dementia Rating (CDR): current version and scoring rules. Neurology 43, 2412-2414. doi: 10.1212/WNL.43.11.2412-a

Morris, J. C., Mohs, R. C., Rogers, H., Fillenbaum, G., and Heyman, A. (1988). Consortium to establish a registry for Alzheimer's disease (CERAD) clinical and neuropsychological assessment of Alzheimer's disease. Psychopharmacol. Bull. $24,641-652$.

Nater, U. M., Hoppmann, C., and Klumb, P. L. (2010). Neuroticism and conscientiousness are associated with cortisol diurnal profiles in adultsrole of positive and negative affect. Psychoneuroendocrinology 35, 1573-1577. doi: 10.1016/j.psyneuen.2010.02.017

Odeniyi, I. A., Fasanmade, O. A., Ogbera, A. O., and Ohwovoriole, A. E. (2015). Body mass index and its effect on serum cortisol level. Niger. J. Clin. Pract. 18, 194-197. doi: 10.4103/1119-3077.151040

Parent-Lamarche, A., and Marchand, A. (2015). The moderating role of personality traits in the relationship between work and salivary cortisol: a cross-sectional study of 401 employees in 34 Canadian companies. BMC Psychol. 3:45. doi: 10.1186/s40359-015-0102-3 
Popp, J., Schaper, K., Kolsch, H., Cvetanovska, G., Rommel, F., Klingmuller, D., et al. (2009). CSF cortisol in Alzheimer's disease and mild cognitive impairment. Neurobiol. Aging 30, 498-500. doi: 10.1016/j.neurobiolaging.2007. 07.007

Popp, J., Wolfsgruber, S., Heuser, I., Peters, O., Hull, M., Schroder, J., et al. (2015). Cerebrospinal fluid cortisol and clinical disease progression in MCI and dementia of Alzheimer's type. Neurobiol. Aging 36, 601-607. doi: 10.1016/ j.neurobiolaging.2014.10.031

Portella, M. J., Harmer, C. J., Flint, J., Cowen, P., and Goodwin, G. M. (2005). Enhanced early morning salivary cortisol in neuroticism. Am. J. Psychiatry 162, 807-809. doi: 10.1176/appi.ajp.162.4.807

Preisig, M., Fenton, B. T., Matthey, M. L., Berney, A., and Ferrero, F. (1999). Diagnostic interview for genetic studies (DIGS): inter-rater and test-retest reliability of the French version. Eur. Arch. Psychiatry Clin. Neurosci. 249, 174-179. doi: 10.1007/s004060050084

Preisig, M., Waeber, G., Vollenweider, P., Bovet, P., Rothen, S., Vandeleur, C., et al. (2009). The PsyCoLaus study: methodology and characteristics of the sample of a population-based survey on psychiatric disorders and their association with genetic and cardiovascular risk factors. BMC Psychiatry 9:9. doi: 10.1186/1471244X-9-9

Pruessner, J. C., Kirschbaum, C., Meinlschmid, G., and Hellhammer, D. H. (2003). Two formulas for computation of the area under the curve represent measures of total hormone concentration versus time-dependent change. Psychoneuroendocrinology 28, 916-931. doi: 10.1016/S0306-4530(02)00108-7

Sapolsky, R. M. (2000). Glucocorticoids and hippocampal atrophy in neuropsychiatric disorders. Arch. Gen. Psychiatry 57, 925-935. doi: 10.1001/archpsyc.57.10.925

Schommer, N. C., Kudielka, B. M., Hellhammer, D. H., and Kirschbaum, C. (1999). No evidence for a close relationship between personality traits and circadian cortisol rhythm or a single cortisol stress response. Psychol. Rep. 84(3 Pt 1), 840-842. doi: $10.2466 / \mathrm{pr} 0.1999 .84 .3 .840$

Sobel, M. (1982). "Asymptotic confidence intervals for indirect effects in structural equation models," in Sociological Methodology, ed. S. Leinhardt (Washington DC: American Sociological Association), 290-312. doi: 10.2307/270723

Stroop, J. R. (1935). Studies of interference in serial verbal reactions. J. Exp. Psychol. 18, 643-662. doi: 10.1037/h0054651

Tatomir, A., Micu, C., and Crivii, C. (2014). The impact of stress and glucocorticoids on memory. Clujul Med. 87, 3-6. doi: 10.15386/cjm.2014.8872. 871. at $1 \mathrm{~cm} 2$

Terracciano, A., Sutin, A. R., An, Y., O’Brien, R. J., Ferrucci, L., Zonderman, A. B., et al. (2014). Personality and risk of Alzheimer's disease: new data and meta-analysis. Alzheimers Dement. 10, 179-186. doi: 10.1016/j.jalz.2013.03.002
Thomas-Antérion, C., Ribas, C., Honoré-Masson, S., Berne, G., Ruel, J. H., and Laurent, B. (2003). Le questionnaire de plainte cognitive (QPC): un outil de recherche de plainte suspecte d'évoquer une maladie d'Alzheimer? Année Gérontol. 17, 56-65.

van Eck, M., Berkhof, H., Nicolson, N., and Sulon, J. (1996). The effects of perceived stress, traits, mood states, and stressful daily events on salivary cortisol. Psychosom. Med. 58, 447-458. doi: 10.1097/00006842-19960900000007

van Santen, A., Vreeburg, S. A., Van der Does, A. J., Spinhoven, P., Zitman, F. G., and Penninx, B. W. (2011). Psychological traits and the cortisol awakening response: results from the Netherlands study of depression and anxiety. Psychoneuroendocrinology 36, 240-248. doi: 10.1016/j.psyneuen.2010.07.014

Vogel, S., Fernandez, G., Joels, M., and Schwabe, L. (2016). Cognitive adaptation under stress: a case for the mineralocorticoid receptor. Trends Cogn. Sci. 20, 192-203. doi: 10.1016/j.tics.2015.12.003

von Gunten, A., Pocnet, C., and Rossier, J. (2009). The impact of personality characteristics on the clinical expression in neurodegenerative disorders-a review. Brain Res. Bull. 80, 179-191. doi: 10.1016/j.brainresbull.2009.07.004

Wilson, R. S., Fleischman, D. A., Myers, R. A., Bennett, D. A., Bienias, J. L., Gilley, D. W., et al. (2004). Premorbid proneness to distress and episodic memory impairment in Alzheimer's disease. J. Neurol. Neurosurg. Psychiatry 75 , 191-195.

Yoshino, A., Kimura, Y., Yoshida, T., Takahashi, Y., and Nomura, S. (2005). Relationships between temperament dimensions in personality and unconscious emotional responses. Biol. Psychiatry 57, 1-6. doi: 10.1016/j. biopsych.2004.09.027

Conflict of Interest Statement: The authors declare that the research was conducted in the absence of any commercial or financial relationships that could be construed as a potential conflict of interest.

The reviewer XZ and handling Editor declared their shared affiliation, and the handling Editor states that the process nevertheless met the standards of a fair and objective review.

Copyright (C) 2017 Ouanes, Castelao, von Gunten, Vidal, Preisig and Popp. This is an open-access article distributed under the terms of the Creative Commons Attribution License (CC BY). The use, distribution or reproduction in other forums is permitted, provided the original author(s) or licensor are credited and that the original publication in this journal is cited, in accordance with accepted academic practice. No use, distribution or reproduction is permitted which does not comply with these terms. 